\title{
The groin area: the Bermuda triangle of sports medicine?
}

Mario Bizzini ${ }^{1}, 2$

The Bermuda triangle, a triangular area in the Atlantic Ocean, is famously known as an area where ships, planes and people have mysteriously disappeared.

The groin, an anatomical region where diagnosis and symptoms are often confusing, may also represent a Bermuda triangle, for clinicians to disappear in vortices of suppositions and assumptions.

Patients with pain in the hip region or groin, typically individuals with a long history of pain and symptoms in this area, are not so easy to manage in clinical sports medicine and physiotherapy practices. There are many common causes of groin pain in athletes with similar signs and symptoms, highlighting the importance, and at the same time the difficulty, of the differential diagnosis. ${ }^{1}$

When treating patients, clinicians often rely on specific knowledge, experience and empirical evidence in clinicaldecision-making. However, according to Sackett's definition, ${ }^{2}$ the latest scientific rehabilitation evidence should also be considered. In sports physiotherapy, leading journals and international conferences may help a clinician to update knowledge and skills. Additionally, consulting evidence databases is one easy way to access research evidence: PEDro, as an example, has offered free access to randomised controlled trials and systematic reviews of physiotherapy interventions for more than 10 years $(11 \%$ of the sports related trials have been published in BJSM). ${ }^{3}$ Note that PEDro now publishes one systematic review in every $B J S M$ issue!

When beginning an examination with a patient who has hip or groin pain, we first gather information related to the history of the problems. Patient-related questionnaires, with proven scientific psychometric quality (validity, reliability,

${ }^{1}$ FIFA-Medical Assessment \& Research Centre, Schulthess Clinic, Zürich, Switzerland ${ }^{2}$ Swiss Sports Physiotherapy Association, Zürich, Switzerland

Correspondence to Dr Mario Bizzini, Schulthess Clinic, Lengghalde 2, Zürich, Switzerland; mario.bizzini@kws.ch responsiveness) are, when available, an important tool in understanding the symptoms and functional limitations of the injured athlete. Thorborg et al (see page 10) recently performed a systematic review of hip and groin disability outcome instruments: only one (low-quality) questionnaire evaluating groin disability was found. This is clearly in contrast to the number of available instruments for the hip joint (dysfunctions, osteoarthritis, arthroplasty, arthroscopy), and illustrates the need for scientific research in this field.

The clinical examination includes inspection, range of movement assessment, various tests for muscle length and strength, specific tests for symptom reproduction (as an example, the resisted test on adductors) and others. Imaging examinations (such as x-rays or MRI) usually complete the examination. The study by Weir et al (see page 6) shows how eight radiological signs of femoroacetabular impingement (FAI) correlate poorly with decreased range of motion (hip rotations) and a specific anterior hip impingement test in patients with longstanding adductor-related groin pain (which, again, is one of the proposed typical presentations of an athlete with an FAI pathology). ${ }^{6}$ Therefore, as we are aware of possible hip joint pathology as a cause of groin pain, we should consider carefully different clinical examinations and their interpretation.

Assessment of hip muscle function with the use of a handheld dynamometer (HHD) is valid, reliable and easy to integrate into routine clinical examinations. ${ }^{7}$ A Danish research group ${ }^{8}$ recently studied (using HHD) the side-to-side eccentric hip adduction and abduction strength symmetry in elite soccer players and matched controls. The interesting results (greater eccentric strength on the dominant side) of this study may find application in the rehabilitation and prevention of adductor muscle injuries in soccer players. No difference in hip adduction strength was found between athletes and controls, but this finding has to be considered within the limitation of the study design (small sample size, no match for training). However, this finding underscores the importance of a differentiated muscle function assessment (an area where there is still a lack of research).

Recently, Choi et al (see page 57) performed a systematic review on the treatment of osteitis pubis (and osteomyelitis) in athletes. The difficulty in the differential diagnosis in athletes presenting with groin pain is apparent. There is a clear lack of evidence for both conservative and operative treatments, as well as a lack of defined criteria for a return to sports. In mild cases of osteitis pubis, there is some evidence for a conservative rehabilitation programme. The additional use of compression shorts and the intake of non-steroidal anti-inflammatory drugs can play a role in the pain and symptom management of osteitis pubis. However, in one reviewed study, more than $20 \%$ of the athletes treated with corticosteroid injections did not respond to this treatment and were unable to return to sport!

\section{Conferences in 2011}

If you are interested in the hip-groin area, and you are looking for the latest evidence in rehabilitation and prevention, you should not miss the IOC World Conference on the Prevention of Injury and Illness in Sports (actually the 3rd World Injury Prevention Congress, after Oslo 2005 and Tromso 2008), which will be held in Monaco in April 2011. It should be noted that the specific symposium on groin injuries is only one of the many highlights in a great 3 -day congress programme (www.ioc-preventionconference.org).

Also, if you are in Switzerland in Autumn (Bern, 18 November 2011), why not attend the 9th Symposium of the Swiss Sports Physiotherapy Association, which will discuss topics related to hip and groin in sports. Dr Tim Hewett (Sports Medicine Biodynamics Center, Cincinnati) and Dr Erik Witvrouw (Ghent University, Belgium) will be among the invited speakers (www.sportfisio.ch).

Provenance and peer review Commissioned; not externally peer reviewed.

Competing interests None.

Accepted 18 November 2010

Br J Sports Med 2011;45:1. doi:10.1136/bjsm.2010.081828

The reference list is published online only at http://bjsm. bmj.com/content/45/1 